\title{
Experiment Research on Initiation and Micro-propagation of the Interface Crack in the Hard Cladding Material 5Cr2Ni08C/Q235
}

\author{
YANG Junru $^{1, \mathrm{a} *}$, WANG Minglan ${ }^{1, \mathrm{~b}}$, WANG Xingfa ${ }^{1, \mathrm{c}}$ and LIU Shu ${ }^{1, \mathrm{~d}}$ \\ ${ }^{1}$ College of Mechanical and Electronic Engineering, Shandong University of Science and Technology, \\ Qingdao, 266590, China

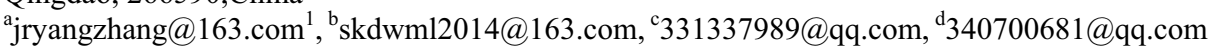

\begin{abstract}
The fracture samples of the hard cladding material $5 \mathrm{Cr} 2 \mathrm{Ni} 08 \mathrm{C} / \mathrm{Q} 235$ are fabricated with the vacuum liquid phase sintering technology, three-point bending fracture experiments are conducted, the sample fracture morphology are observed with SEM. The experiment research results show that: the intergranular micro-crack is easy to initiate in the interface under the action of the external load, and the micro-crack easily appears on the crystal boundary, the thermal expansion coefficient mismatch places, around the interface contamination and micro holes. Based on which, the interface micro-crack easily continues to propagate along the crystal boundary. And the micro-mechanisms of the interface crack initiation and propagation are established.
\end{abstract}

\section{Introduction}

Ternary boride hard cladding material is a new kind of cladding material with good comprehensive performances of high wear resistance, corrosion resistance, fatigue resistance, the chemistry stability, heat resistance and so on. It has been widely used in the fields of aerospace, automobile, nuclear industry. Because of the material property mismatch between two sides of the interface, the micro-crack is easy to initiate and propagate. The interface cracks have serious influence on the performance and usage of the cladding material.

Up to now, the researches have achieved some achievements in micro-mechanism of the interface crack initiation and propagation in the composite material. Jin Yunxue et al [1] researched the crack propagation in the $\mathrm{TiC} / \mathrm{Ti}$ composite material under the action of dynamic tensile load, which showed that the micro-crack was easy to initiate on TiC particle surface and at the stress concentration position. Liu Xiaoyu [2] made experiment research on the deformation and fracture of the functionally graded material, the result showed that the strength between the composite layers was insufficient, the crack usually generated in the composite layer extended along the loading direction, and deflected along the interface. S.A.Nazarov et al [3] used energy release rate method to study the crack propagation in two kinds of elastic heterogeneous composite material. Hu Tuo [4] used finite element numerical simulation method to study the mechanism of the interface crack

* Corresponding author: jryangzhang@163.com 
propagation in the composite materials, the result showed that the interface crack in the composite pressure vessel appeared elliptical propagation and propagated to the material with small elastic modulus. R. Li et al [5] researched the influence of the thermal load on the interface crack branched propagation in anisotropic elastic material using numerical simulation method. Yu Hanghai et al [6] studied the resistance force of crack propagation in $\mathrm{BNNT} / \mathrm{Si}_{3} \mathrm{~N}_{4}$ composites with the finite element method. Li Wei et al [7] studied the fatigue crack extension in the spray deposition of $\mathrm{SiCp} / \mathrm{Al}-7 \mathrm{Si}$ composite, the result showed that the crack tip would deflect when it met the reinforced particle.

The initiation and propagation of the micro-crack in some different composite material have been studied respectively. As the new composite material, the hard cladding material $5 \mathrm{Cr} 2 \mathrm{Ni} 08 \mathrm{C} / \mathrm{Q} 235$ is less researched on its micro-mechanism of the interface crack initiation and propagation. In the paper, the experiment researches on the interface crack initiation and propagation with three point bending test method are carried out. The research results will play an important role to reveal the essence of the interface micro-crack initiation and extension in the composites and to the optimization design of cladding material parts.

\section{Experimental Scheme}

Sample Fabrication. The substrate material is Q235 metal sheet. The cladding raw materials contain powdered alloy ( $\mathrm{FeB}, \mathrm{Ni}, \mathrm{Mo}$ powder, carbonyl iron), carbon powder, binder and the solvent etc. Mix these cladding raw materials according to certain proportion to make homogeneously dispersed and stable slurry. Spray the slurry on Q235 steel substrate through spray forming technology, after drying, using the vacuum liquid phase sintering technology to make the hard cladding material 5Cr2Ni08C/Q235, the technology process [8-11] is shown in Fig.1.

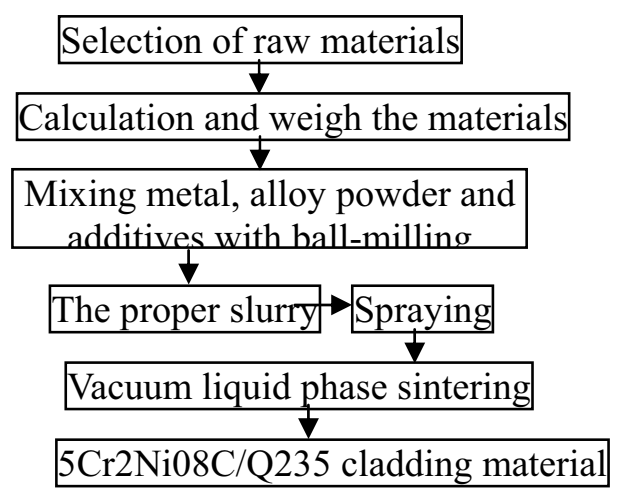

Fig.1 Technology process of the cladding material $5 \mathrm{Cr} 2 \mathrm{Ni} 08 \mathrm{C} / \mathrm{Q} 235$
The prefabricated crack

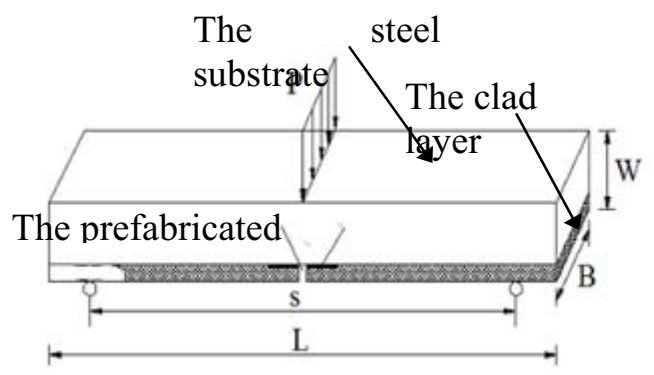

Fig. 2 Fracture sample

After grinding and polishing the cladding material $5 \mathrm{Cr} 2 \mathrm{Ni08C} / \mathrm{Q} 235$, the sample is gotten by linear cutting, and its size is $B \times W \times L=3 \mathrm{~mm} \times 4 \mathrm{~mm} \times 36 \mathrm{~mm}$ ( $B$ is the sample width, $\mathrm{W}$ is the sample thickness, $\mathrm{L}$ is the sample length), and its span $\mathrm{S}$ is $20 \mathrm{~mm}$. Cut a gap with the width of $0.25 \mathrm{~mm}$ in the middle position of the sample through the clad layer thickness. Then prefabricate a crack parallel with and lying on the interface at the notch root. The fracture sample is shown in Fig.2.

Experiment Process. As the hard cladding material is brittle, the bending strength for the clad layer under the action of tensile stress is lower than that under the action of compression stress [8]. Therefore, the bending fracture test is made under the former condition. The three-point bending test is operated on the DBSL-SJ-30t electronic universal 
testing machine. According to the experimental purpose and requirement to add load on the sample, the load frequency is fixed, the initial force is set to $30 \mathrm{~N}$, increase the load continuously and slowly, the movement speed of the pressure head is $2 \mathrm{~mm} / \mathrm{min}$, until the samples fracture. Use the KYKY-2800B scanning electron microscope (SEM) to observe the fracture morphology to analyze the interface crack initiation and propagation mechanism.

\section{Experiment Results and Analyses.}

Fracture Morphology of the Sample. Fig.3 shows the sample fracture morphology. The upper layer with group of protruding particles and bright color is the clad layer, the middle hollow part is the interface, the lower and relatively smooth part is the steel substrate.

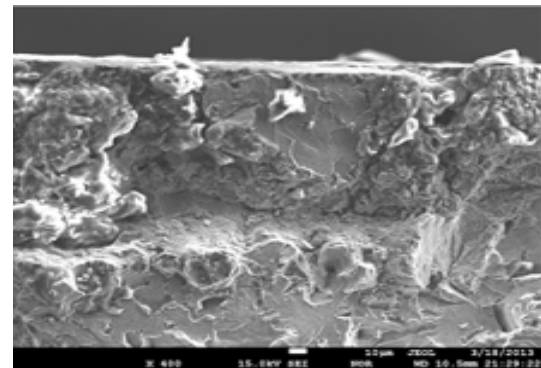

Fig.3 Sample fracture morphology

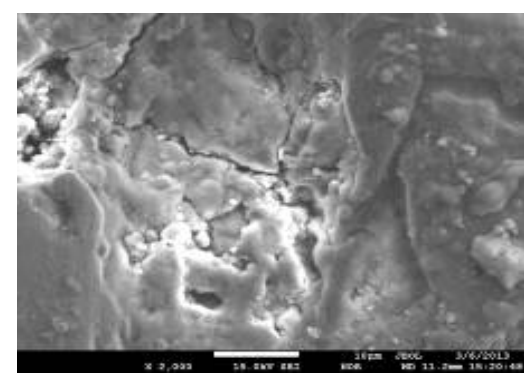

Fig. 4 The interface microstructure morphology

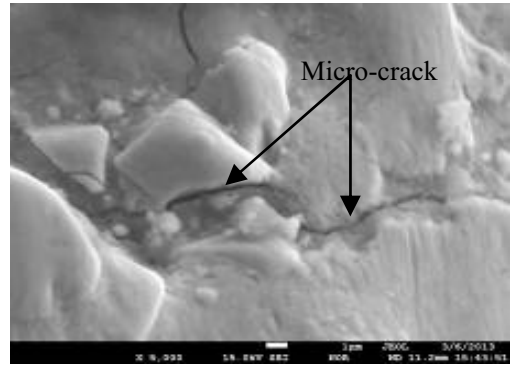

Fig. 5 SEM micrograph of intergranular fracture

Fig. 4 shows the interface microstructure morphology. The middle part with white light is the interface, the right area of the interface with deep color is the steel substrate, the left area of the interface is the clad layer. It can be seen that the interface has coarse grains and defects such as contamination, micro cracks, micro holes, and that the micro cracks perpendicular to and parallel with the interface are distributed along the crystal boundary.

\subsection{Initiation Mechanism Analyses of the Interface Micro-crack.}

Interface Micro-crack Initiation along the Crystal Boundary. Fig.5 is SEM micrograph of intergranular fracture. The coarse and highlighted grains show that the intergranular fracture takes place, the micro cracks initiate at the crystal boundary. Therefore, the interface micro-cracks easily initiate along the crystal boundary.

The Micro-crack Initiation Near the Thermal Expansion Coefficient Mismatch Place. Fig.6 shows the micro-crack initiation near the thermal expansion coefficient 
mismatch place. The right area with black color is the steel substrate, the left side with bright white region is the clad layer, the transition zone between the clad layer and steel substrate is the interface. It is obvious that a wide micro-crack initiates in the interface. The material property mismatch between two sides of the interface results in the residual stress concentration, which causes the interface micro cracks initiation. Micro cracks under the action of external load become wider and extend to form a main crack along the interface of thermal expansion mismatch.

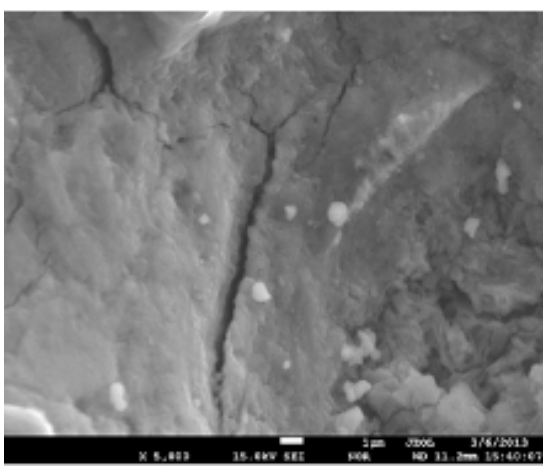

Fig.6 The micro-crack initiation near the thermal expansion coefficient mismatch place

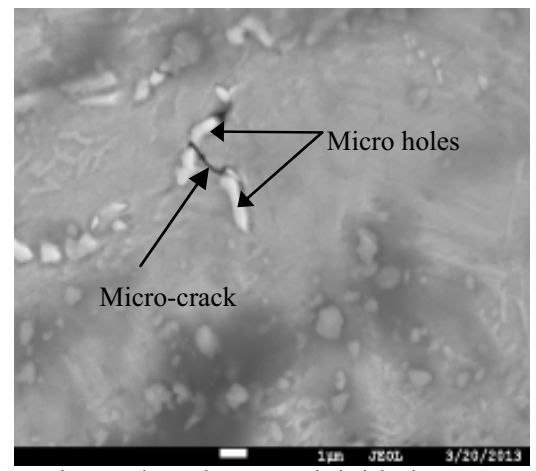

Fig. 7 The micro-crack initiation near the micro holes

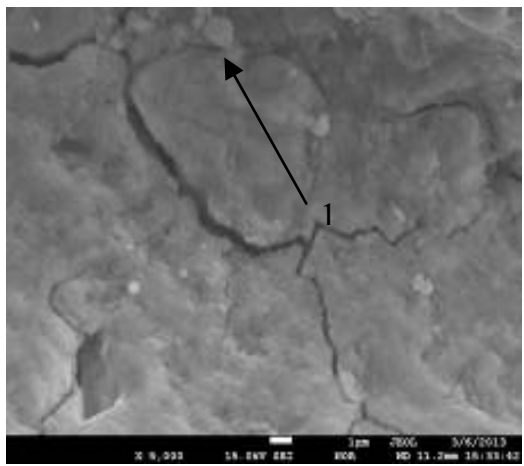

Fig. 8 The micro-crack initiation near the carbide

The Micro-crack Initiation Near the Micro Holes. Fig.7 shows the micro-crack initiation near the micro holes. It shows that the micro holes appear in the interface between hard phase grains, micro holes become larger under the action of external load and form the micro-crack in the end.

The Micro-crack Initiation Near the Carbide. Fig.8 shows the micro-crack initiation near the carbide. When the dislocation caused by the external force encounters the carbide, if the carbide is not cut apart, it will lead to the dislocation pile-up at other phase interface and stress concentration. Finally the carbide will separate from the combination surface, which causes the micro-crack initiation around the carbide (as shown in Fig.8 mark 1). A large number of carbides at grain boundary change the material property of the grain boundary and the near areas, when adjacent grain boundary cannot coordinate their deformation, micro cracks initiate along crystal boundary.

Overall, the experiment results show that, in the hard cladding material $5 \mathrm{Cr} 2 \mathrm{Ni} 08 \mathrm{C} / \mathrm{Q} 235$, the interface micro-crack is easy to initiate on the grain boundary, the 
thermal expansion coefficient mismatch places, around the interface contamination and micro holes.

\subsection{Propagation Mechanism Analyses of the Interface Micro-crack.}

Intergranular Propagation of the Interface Micro-crack. Fig.9 shows the intergranular propagation of the interface micro-crack. It can be seen that, during the interface micro-crack propagation process, there are a lot of crack deflection and branching, and obvious cracking on the grain boundary (Fig.9 (a) position "1"). In Fig.9 (b), when the micro-crack expands to the grain tip, it does not run through the grain, but continues to expand along the crystal boundary on the triple grain boundary junction. Therefore, the interface micro-crack mainly takes place intergranular propagation.

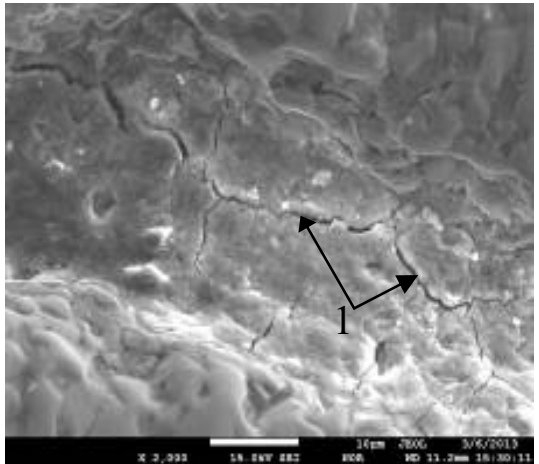

(a)

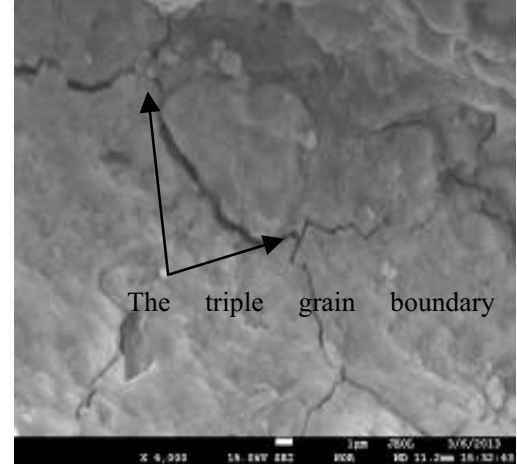

(b)

Fig. 9 The intergranular propagation of the interface micro-crack

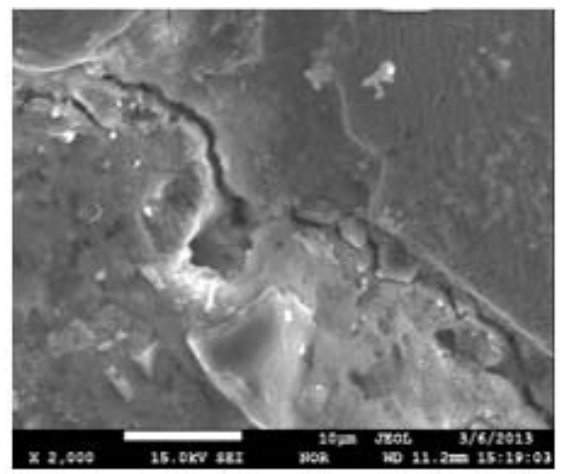

Fig. 10 The interface micro-crack propagation near the substrate

Fig.10 shows the interface micro-crack propagation near the substrate. The upper right gray area is the steel substrate, the lower left bright area is the interface. It is shown in the figure that the micro-crack is distributed along the crystal boundary. Fig.11 shows the interface micro-crack propagation near the clad layer. The white blob at the upper area is the clad layer, and the interface is at the lower half needle-like area with black and white. From the figure, it is also obvious that the micro-crack is distributed along the crystal boundary, i.e. the micro-crack propagates along the crystal boundary.

Therefore, from the experiment result, there is main intergranular propagation of the 
interface micro-crack in the cladding material.

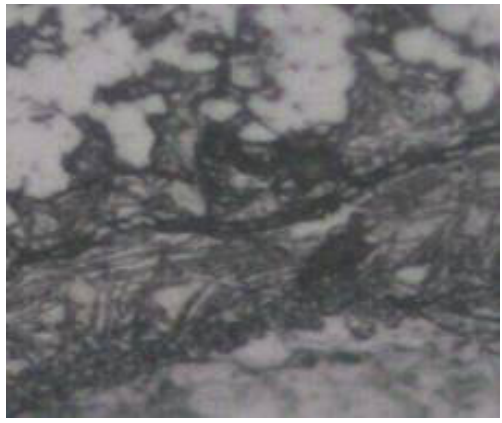

Fig.11 The interface micro-crack propagation near the clad layer

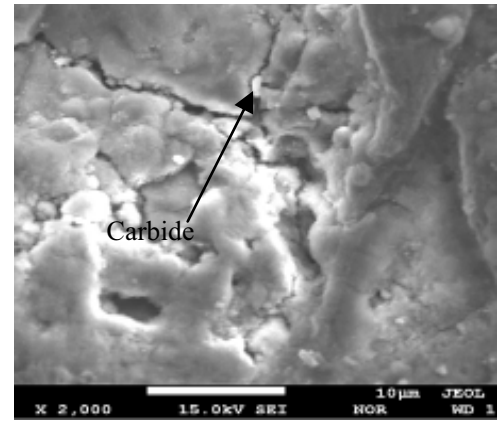

Fig.12 Effects of the carbide on the micro-crack propagation

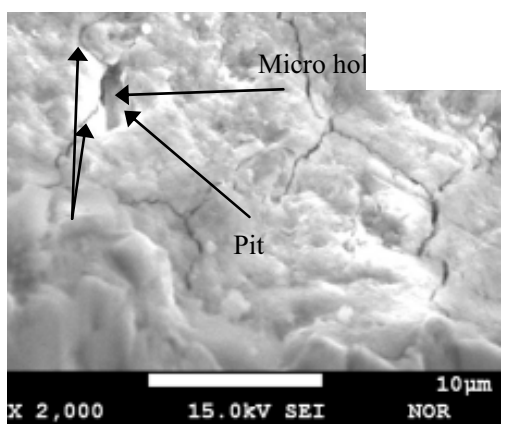

Fig.13 Effects of micro holes on the micro-crack propagation

Effects of the Carbide on the Micro-crack Propagation. Fig.12 shows the carbide effects on the micro-crack propagation. When the micro-crack meets the carbide, it strides across the carbide and propagates on both sides of the carbide. The carbide like "bridge" hinders the propagation of the micro-crack. With the load increase, the micro-crack gradually becomes bigger and continues to propagate along one side of the carbide.

Effects of Micro Holes on the Micro-crack Propagation. Fig. 13 shows the micro-crack propagation near the micro holes. Under the action of the external load, the micro-crack initiated on the micro holes propagates slowly by connecting with the micro holes generated in front of the crack tip or micro-gap on the edge of the micro holes. With the increase of external load, the micro-crack continues to propagate from the micro-gap. When the micro holes reach a certain size, the interface hard phase starts to peel off which causes a pit in the interface. With the energy increase, the micro holes become bigger to continually propagate along the weak part in the interface. When the micro-crack propagation meets micro holes, the crack continues to expand along one side of the hole without crossing it.

Overall, the experiment results show that, in hard cladding material $5 \mathrm{Cr} 2 \mathrm{Ni} 08 \mathrm{C} / \mathrm{Q} 235$, the interface micro-crack mainly takes place intergranular propagation. When the micro-crack meets the carbide, it strides across the carbide and propagates on both sides of the carbide. When the micro-crack propagation meets micro holes, the crack continues to expand along one side of the hole without crossing it. 


\section{Conclusion}

In this paper, the experiment researches with the three-point bending fracture test to the hard cladding materials $5 \mathrm{Cr} 2 \mathrm{Ni} 08 \mathrm{C} / \mathrm{Q} 235$ fracture sample are carried out. The results show that the micro-mechanisms of the interface crack initiation and propagation in the hard cladding material are as the following: the intergranular micro-crack is easy to initiate in the interface, which easily appears on the crystal boundary, the thermal expansion coefficient mismatch places, around the interface contamination and micro holes; the interface micro-crack mainly takes place intergranular propagation, when micro-crack propagation meets the micro holes or the carbide, it continues to expand along one side of the hole or carbide without crossing them finally.

\section{Acknowledgements}

This project is supported by the natural science foundation of Shandong Province (ZR2013EEM016), and postgraduate innovation fund project of Shandong University of Science and Technology (YC150210).

\section{References}

1. Jin Yunxue, Li Jungang, Formation and propagation mechanisms of dynamic tensile cracks in $\mathrm{TiC} / \mathrm{Ti}$ composite, Rare Metal Materials and Engineering. 36 (2007) 764-768.

2. Liu Xiaoyu, Study on deformation and fracture of $\mathrm{SiC} / \mathrm{Al}$ functionally gradient materials, Ph.D. thesis, Tsinghua University, Beijing, China, 2013.

3. S.A.Nazarov, M.Specovius-Neugebauer, M.Steigemann, Crack propagation in anisotropic composite structures, Asymptotic Analysis. 86 (2014) 123-153.

4. Hu Tuo, The numerical simulation on composite materials interface crack propagation mechanism, Ph.D. thesis, Nanchang University, Nanchang, China, 2013.

5. R. Li, George A. Kardomateas, A solution to the thermo-elastic interface crack branching in dissimilar anisotropic bi-material media, International Journal of Solids and Structures. 43(2006) 913-942.

6. Yu Hanghai, Yang Jun, Liu Nana, Zhang Yongting, Zheng Chunying, Finite element analysis of the crack propagation of $\mathrm{Si}_{3} \mathrm{~N}_{4}$ composites reinforced by $\mathrm{BN}$ nanotubes, Shandong Product Quality Inspection Research Institute. 23 (2014) 85-88.

7. Li Wei, Chen Zhenhua, Chen Ding, Teng Jie, Growth behavior of fatigue crack in spray-formed SiCp/Al-7Si composite, Acta Metallurgica Sinica. 47 (2011) 102-108.

8. Yang Junru, Study on theory and reliability of cermet hard cladding parts, Ph.D. thesis, Shandong University, Jinan, China, 2006.

9. Wang Yongguo, Cao Yuan, Li Zhaoqian, Zhang Tao, Analysis for the micro-structure of the hard coating material, Journal of Electron Microscopy. 21 (2002) 461-465.

10. Liu Futian, Study on wear resistance and corrosion resistance of cermet hard cladding materials, Ph.D. thesis, Shandong University, Jinan, China, 2003.

11. Dong Fei, Liu Futian, Study on hard alloy cladding-steel substrate interface bonding layer, Materials and Applications. 7 (2011) 74-77. 